clearly illustrated by the redundancy in organizational structure of the stress response systems in the CNS and the skin $(8,13)$. This redundancy has its functional consequences when psychological stress-mediated activation of central HPA axis signaling negatively affects protective and antimicrobial skin barrier function, as is demonstrated in this issue by Aberg et al. (7). These findings will hopefully have clinical impact by stimulating the development of systemic and topical selective receptor antagonists for peptide and steroidal messengers of the HPA axis, as well as topical use of stimulators of cortisol metabolic inactivation, and the use of inhibitors of steroidogenesis in order to improve the antimicrobial and protective barrier activity of the skin. This may also be the dawn of a new clinical approach for the treatment of psychological stress-induced inflammatory dermatoses via the use of systemic or topical CRF1 antagonists.

\section{Acknowledgments}

Comments from Jacobo Wortsman and Curtis Dohan as well as NIH support (grant AR052190) are acknowledged.

Address correspondence to: Andrzej Slominski, Department of Pathology and
Laboratory Medicine, Health Science Center, University of Tennessee, 930 Madison Avenue, Memphis, Tennessee 38163, USA. Phone: (901) 448-3741; Fax: (901) 4486979; E-mail: aslominski@utmem.edu.

1. Elias, P.M. 2005. Stratum corneum defensive functions: an integrated view. J. Invest. Dermatol. 125:183-200.

2. Arck, P.C., Slominski, A., Theoharides, T.C., Peters, E.M., and Paus, R. 2006. Neuroimmunology of stress: skin takes center stage. J. Invest. Dermatol. 126:1697-1704.

3. McEwen, B.S. 2007. Physiology and neurobiology of stress and adaptation: central role of the brain. Physiol. Rev. 87:873-904.

4. Chrousos, G.P. 1995. The hypothalamic-pituitaryadrenal axis and immune-mediated inflammation. N. Engl. J. Med. 332:1351-1362.

5. Vale, W., Spiess, J., Rivier, C., and Rivier, J. 1981. Characterization of a 41-residue ovine hypothalamic peptide that stimulates secretion of corticotropin and beta-endorphin. Science. 213:1394-1397.

6. Turnbull, A.V., and Rivier, C. 1995. Regulation of the HPA axis by cytokines. Brain Behav. Immun. 9:253-275.

7. Aberg, K.M., et al. 2007. Psychological stress downregulates epidermal antimicrobial peptide expression and increases severity of cutaneous infections in mice. J. Clin. Invest. 117:3339-3349. doi:10.1172/ JCI31726.

8. Slominski, A., and Wortsman, J. 2000. Neuroendocrinology of the skin. Endocr. Rev. 21:457-487.

9. Stenn, K.S., and Paus, R. 2001. Controls of hair follicle cycling. Physiol. Rev. 81:449-494.

10. Paus, R., Theoharides, T.C., and Arck, P.C. 2006 Neuroimmunoendocrine circuitry of the 'brain- skin connection'. Trends Immunol. 27:32-39.

11. Slominski, A., Wortsman, J., Luger, T., Paus, R., and Solomon, S. 2000. Corticotropin releasing hormone and proopiomelanocortin involvement in the cutaneous response to stress. Physiol. Rev. 80:979-1020.

12. Slominski, A., et al. 2006. Corticotropin releasing hormone and the skin. Front. Biosci. 11:2230-2248.

13. Slominski, A., Wortsman, J., Tuckey, R.C., and Paus, R. 2007. Differential expression of HPA axis homolog in the skin. Mol. Cell. Endocrinol. 265-266:143-149.

14. Slominski, A., et al. 2004. A novel pathway for sequential transformation of 7-dehydrocholesterol and expression of the P450scc system in mammalian skin. Eur. J. Biochem. 271:4178-4188.

15. Slominski, A., Tobin, D.J., Shibahara, S., and Wortsman, J. 2004. Melanin pigmentation in mammalian skin and its hormonal regulation. Physiol. Rev. 84:1155-1228.

16. Zbytek, B., Wortsman, J., and Slominski, A. 2006. Characterization of a ultraviolet B-induced corticotropin-releasing hormone-proopiomelanocortin system in human melanocytes. Mol. Endocrinol. 20:2539-2547.

17. Ito, N., et al. 2005. Human hair follicles display a functional equivalent of the hypothalamic-pituitary-adrenal axis and synthesize cortisol. FASEB J. 19:1332-1334.

18. Zbytek, B., and Slominski, A.T. 2007. CRH mediates inflammation induced by lipopolysaccharide in human adult epidermal keratinocytes. J. Invest. Dermatol. 127:730-732.

19. Luger, T., Paus, R., Lipton, J., and Slominski, A. 1999. Cutaneous neuromodulation: the proopiomelanocortin System. Ann. N. Y. Acad. Sci. 885:1-479.

20. Bohm, M., Luger, T.A., Tobin, D.J., and Garcia-Borron, J.C. 2006. Melanocortin receptor ligands: new horizons for skin biology and clinical dermatology. J. Invest. Dermatol. 126:1966-1975.

\title{
OX40 signaling directly triggers the antitumor effects of NKT cells
}

\author{
Dapeng Zhou
}

Department of Melanoma Medical Oncology, Division of Cancer Medicine, University of Texas MD Anderson Cancer Center, Houston, Texas, USA.

\begin{abstract}
Pathways involving the costimulatory molecule OX40 and OX40 ligand $(\mathrm{OX} 40 \mathrm{~L})$ enhance tumor rejection. It was presumed that this effect was mediated by changes in DCs and/or T cells. In this issue of the JCI, Zaini et al. report that, in mice, intratumoral injection of DCs genetically modified to express OX40L suppressed the growth of a preexisting melanoma by directly triggering an antitumor NKT cell response (see the related article beginning on page 3330). This work suggests that the intratumoral NKT cell population may be harnessed for cancer immunotherapy and that $\mathrm{OX} 40$ costimulation may be used as a unique trigger of the antitumor activity of these cells.
\end{abstract}

The effective $\mathrm{T}$ cell response to a tumor antigen requires not only stimulation by

Nonstandard abbreviations used: $\alpha$-GalCer, $\alpha$ galactosylceramide; OX40L, OX40 ligand; OX40L-DC, OX40L gene-transduced DC.

Conflict of interest: The author has declared that no conflict of interest exists.

Citation for this article: J. Clin. Invest. 117:3169-3172 (2007). doi:10.1172/JCI33976. the tumor antigen itself via the TCR signaling pathway, but also costimulatory molecules (1). In the early stage of T cell stimulation, the costimulatory molecule CD28 is required for $\mathrm{T}$ cell proliferation and survival through recognition of B7 molecules expressed by DCs. "Second-wave" costimulatory molecules, represented by TNF receptor family members, are required by activated $\mathrm{T}$ cells for completion of their differentiation programs and exertion of their antitumor functions such as the release of cytokines and cytotoxic granules. The TNF receptor family of costimulatory molecules continues to be of interest in an effort to identify candidate targets for the development of immunotherapeutics. Activation of many of these signaling pathways has caused tumor rejection in preclinical and clinical studies (2).

\section{OX40 and OX40 ligand in antitumor immunity}

The OX40 costimulatory molecule is a member of the TNF receptor family and is expressed on activated CD4 ${ }^{+} \mathrm{T}$ cells and $\mathrm{CD}^{+} \mathrm{T}$ cells (1). OX40 ligand (OX40L) belongs to the TNF superfamily and is 
expressed on activated professional antigenpresenting cells such as DCs, macrophages, and B cells. The requirement of OX40 and OX40L in adaptive immune responses, especially in CD4 memory responses, is well established and has been reviewed elsewhere (1). OX40 signaling has been suggested to sustain the signaling pathways of the TCR, CD28, and the IL-2 receptor.

Manipulation of OX40 signaling has been shown to be an efficient approach to enhance antitumor immunity in several preclinical studies (3-5). These therapies include adoptive transfer of tumor-specific $\mathrm{CD}^{+} \mathrm{T}$ cells followed by vaccine therapy (3), combined therapy with an OX40 agonist and other costimulatory agents, and use of an OX40 agonist as an adjuvant for wholetumor cell vaccines (5). The mechanism of action of OX40 in these different tumor systems is quite possibly variable. Expression of $\mathrm{OX} 40$ by $\mathrm{CD}^{+}$and/or $\mathrm{CD}^{+} \mathrm{T}$ cells is essential for OX40 agonists to exert their effect (3-5). DCs, the professional antigenpresenting cells that present tumor antigens, are the critical cellular component required to activate $T$ cells. The role of OX40 in crosstalk between DCs and CD4 ${ }^{+}$ and/or $\mathrm{CD}^{+} \mathrm{T}$ cells in tumor growth is only partially understood. Clearly, the TCR stimulation signal from DCs is the first signal required. It is also very clear that OX40 agonists provided critical signals after initial TCR stimulation. However, many questions remain: What are the exact molecular mechanisms leading to the functional changes of both DCs and T cells? What are the roles of other costimulatory molecules expressed by DCs? Do other immune cells such as macrophages, plasmacytoid DCs, NK cells, and NKT cells play a role in the crosstalk between DCs and T cells?

\section{NKT cells make a major difference}

In this issue of the JCI, Zaini et al. report a critical role for NKT cells in the therapeutic effect of OX40 in mice bearing a preexisting tumor (6). The authors developed a cell therapy method using bone marrow-derived DCs that were genetically engineered to overexpress OX40L through a recombinant adenoviral-transducing approach. The majority of OX40L genetransduced DCs (OX40L-DCs) expressed a 10-fold higher amount of OX40L protein on the cell surface. The function of the OX40L gene in the adenovirally transduced DCs was verified by an immunization challenge experiment. Vaccinating mice by OX40L-DC-pulsed protein vac- cines generated protective $\mathrm{T}$ cell immunity in a tumor prevention model. In order to locate the OX40 interaction to the tumor microenvironment, the authors used an intratumoral injection approach to treat mice carrying preexisting solid tumors. Excitingly, the intratumoral OX40LDC cell therapy caused the rejection of established tumors, including a most aggressive, poorly immunogenic B16F10 melanoma. The therapeutic effects were strictly dependent on OX40 expression in the tumor-bearing mice, because OX40-/mice failed to reject the tumor. Not surprisingly, both $\mathrm{CD}^{+}$and $\mathrm{CD}^{+} \mathrm{T}$ cells were also required.

A major finding reported by Zaini et al. (6) is that NKT cells are essential for the success of the OX40L-DC intratumoral therapy. OX40L-DCs failed to treat tumors in mice lacking NKT cells, and this report very nicely demonstrates the involvement of NKT cells by both gain-of-function and loss-of-function experiments.

Analysis of the lymphocyte population infiltrating the tumors in these animals indicated that $20 \%$ of the OX40-expressing cells were NKT cells (6). To estimate the function of these NKT cells, Zaini et al. used an intracellular staining technology to measure the cytokines they produce. IFN- $\gamma$ was produced by $73 \%$ of the intratumoral NKT cells. Furthermore, a significant increase in IFN- $\gamma$ was found in the tumor lysates. IFN- $\gamma$ is a cytokine with well-established, potent antitumor functions, including sensitization of tumor cells for $\mathrm{CD}^{+} \mathrm{T}$ cell cytotoxicity and induction of angiostatic chemokines (7). Consistent with these findings, a 3 -fold increase in intratumoral infiltration of $\mathrm{CD}^{+}$and $\mathrm{CD}^{+} \mathrm{T}$ cells was observed, suggesting increased production of intratumoral chemokines that may attract $\mathrm{T}$ cells. Future systemic studies on other immune cells and cytokine/chemokines in the tumor microenvironment might complete our understanding of these OX40L-DC-elicited responses. Of note, in human patients, positive associations between NKT cell presence in primary tumors and long-term survival have been demonstrated for distinct cancers (8). It might be interesting to study whether OX40L is associated with the presence of NKT cells.

\section{DC-NKT cell crosstalk and the role of OX40}

Invariant NKT cells represent a very special subset of innate lymphocytes that express markers of both NK cells and T cells. They express a very restricted repertoire of TCRs (V $\alpha 14$ in mice and V $\alpha 24$ in humans). In contrast to conventional $\mathrm{T}$ cells, which recognize peptide antigens presented by MHC molecules, NKT cells recognize glycolipid antigens presented by the nonpolymorphic, non-MHC antigen-presenting molecule CD1d (Figure 1). The activation of conventional $\mathrm{T}$ cells requires more than 12 hours of antigen stimulation, while NKT cells can be activated within 2 hours, a mechanism more similar to NK cells. The antitumor effect of invariant NKT cells was first discovered via mechanistic studies of the effects of the NKT cell stimulant $\alpha$-galactosylceramide $(\alpha$-GalCer), a marine spongederived glycosphingolipid that prevents metastasis of intravenously injected B16 melanoma cells in mice (9). The antitumor effect of NKT cells in established solid tumors is an area of research in which several central questions currently remain unanswered, such as: What is the life cycle of intratumoral NKT cells? What antigenic stimulation and costimulatory signals control the fate of NKT cells? What elements of the immune response (cellular or secreted factors) are involved in NKT cell-induced immune responses, and how? DC-NKT cell interaction is the starting point for us to find answers for these questions.

In this OX40L-DC intratumoral therapy model (6), it is a challenging task to study the crosstalk between DCs and NKT cells. Ideally, experimental systems should be designed to reproduce a scenario similar to the intratumoral interaction between OX40L-DCs and NKT cells. However, technically, this is extremely difficult because the tumor microenvironment contains multiple cellular components (tumor cells, granulocytes, DCs, macrophages, NK cells, $\mathrm{CD}^{+}{ }^{+} \mathrm{T}$ cells, CD8 ${ }^{+} \mathrm{T}$ cells, and other cell types) as well as extremely diverse profiles of cytokines/chemokines produced by these cells. Simply mixing the OX40L-DCs with fresh NKT cells may not work. In view of this, Zaini et al. studied OX40 signaling by stimulating NKT cells with a potent agonist NKT antigen, $\alpha$-GalCer. Both in vivo and in vitro experiments indicated that enhanced IFN- $\gamma$ production is an outcome of OX40 signaling in NKT cells. As expected, in all experiments, NKT cell activation was strictly dependent on TCR stimulation, because the production of 


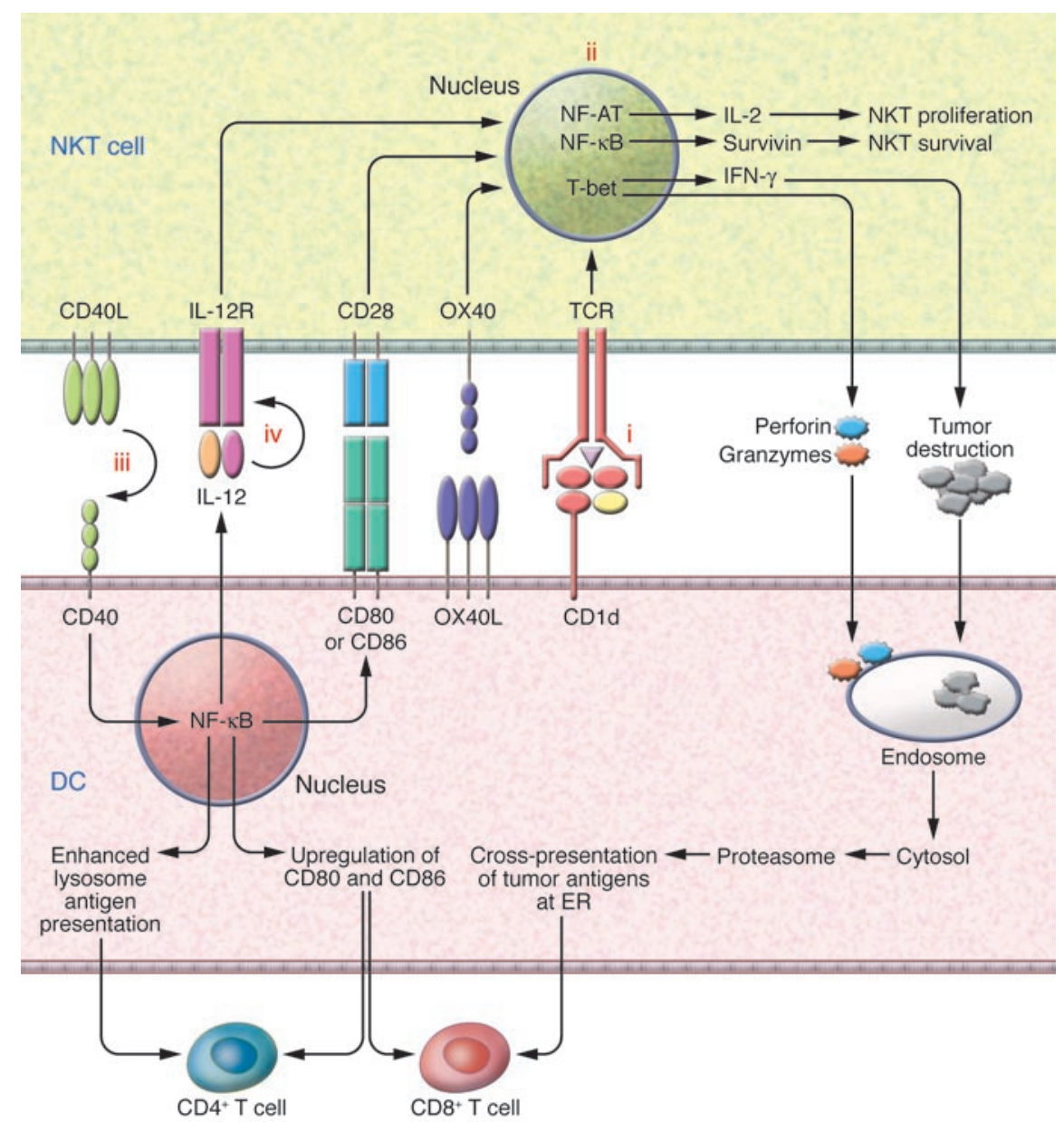

Figure 1

Crosstalk between OX40L-DCs and NKT cells. It is hypothesized that OX40L-DCs first stimulate NKT cells (i) to proliferate and produce cytokines (ii). Subsequently, NKT cells activate DCs through cellular contacts (such as CD40/CD40L binding) and cytokine expression (iii), which induce the DCs to secret IL-12 and to upregulate costimulatory molecules such as CD80 and CD86. IL-12 stimulates the IL-12 receptor expressed by activated NKT cells (iv), which further potentiates NKT cell activation and forms a positive-feedback loop. Three lines of signaling transduction pathways are speculated to be activated in NKT cells: (a) TCR (V $\alpha 14$ in mouse and V $\alpha 24$ in human) signaling is assumed to be the first signaling pathway; (b) other costimulatory signaling networks such as CD28 might be involved and interact with both the TCR signaling network and the OX40 signaling network; and (c) OX40 signaling components may control the fate of NKT cells through molecular interactions with the TCR signaling network. OX40 interacts with TNF receptor-associated factors and may lead to the regulation of transcription factors such as NF-AT, NF-kB, and T-bet.

IFN- $\gamma$ by splenocytes could be completely blocked by anti-CD1d antibody, which blocks the TCR recognition of $\alpha$-GalCer presented by CD1d.

An interesting point in the intratumoral DC-NKT cell interaction system described by Zaini et al. (6) is that the antigens that stimulate NKT cells are endogenous glycolipids presented by DCs, not foreign glycolipids such as $\alpha$-GalCer. It is generally accepted that endogenous, self-glycolipid antigens for NKT cells are weaker antigens compared with $\alpha$-GalCer. As a super agonist antigen, $\alpha$-GalCer might provide stronger TCR signaling, which may partially bypass the costimu- latory pathways that are required by the natural endogenous glycolipid ligands. Thus, in the scenario of TCR stimulation by endogenous ligands, there may be much more dependence on OX40 costimulation, and the defect of NKT cell function observed in $\mathrm{OX} 40^{-/-}$mice may be more severe.

\section{OX40 as a molecular switch at both priming and effector phases}

The antitumor immune response is a multistep process consisting of 2 phases. The first, the antigen-priming phase, involves the processing and presentation of tumorassociated antigens by antigen-presenting cells and the activation and proliferation of antigen-specific T cells. In the second phase, known as the effector phase, activated immune cells travel to the tumor site and exert their effector functions, which include cytokine production.

The critical role of DC-NKT cell crosstalk in the antigen-priming phase to generate tumor-specific $T$ cells was previously reported by Fujii et al. using $\alpha$-GalCer as the NKT stimulus (10) and demonstrated by the immunization challenge experiments of Zaini et al. using OX40 agonists (6). The mechanisms by which NKTs interact with OX40L-DCs in order to enhance the capacity of DCs to activate $\mathrm{CD}^{+}$and 
$\mathrm{CD}^{+} \mathrm{T}$ cells remain unclear. The activation of DCs may involve a positive feedback loop consisting of IL-12 (secreted by DCs) and the IL-12 receptor (expressed by activated NKTs; Figure 1). Activated DCs stimulate $\mathrm{CD}^{+}$and $\mathrm{CD}^{+}{ }^{+} \mathrm{T}$ cells through enhanced antigen presentation and expression of costimulatory molecules such as CD80, CD86, and CD40. To present tumor antigens to $\mathrm{CD}^{+} \mathrm{T}$ cells, a "cross-priming" process is required for the endocytosed tumor antigens to enter the cytosol of DCs before being processed by the proteasome and loaded in the endoplasmic reticulum. NKT cells might enhance the cross-priming process via the secretion of perforin, granzymes, or some as yet unknown molecular components into DCs, and cause the endocytosed tumor antigens to detour to the cytoplasm. Besides cells of the adaptive immune system, innate immune cells might also be critical for mediating the effects of the OX40L-DC treatment. In view of the potent production of IFN- $\gamma$ and perforin by NK cells caused by NKT cell activation, the OX40L-DC treatment may be NK cell dependent.

It should be highlighted that in the effector phase of antitumor immunity, NKT cells, $\mathrm{CD}^{+}$and $\mathrm{CD}^{+} \mathrm{T}$ cells, and NK cells convene to kill tumor cells, and crosstalk of these multiple cells may be essential for tumor rejection. OX40 expression by $\mathrm{CD}^{+}$and $\mathrm{CD}^{+} \mathrm{T}$ cells has been shown to be critical for cooperation between these 2 major cellular elements $(3,11)$. OX 40 expression enhances the differentiation of $\mathrm{CD}^{+} \mathrm{T}$ cells $(12,13)$. OX40 expression on $\mathrm{CD}^{+} \mathrm{T}$ cells, and the presence of an OX40 agonist, control the proliferation and function of $\mathrm{CD}^{+} \mathrm{T}$ cells $(3,11)$. Interestingly, activated NK cells express OX40L, which might initiate crosstalk between NK cells and $\mathrm{CD}^{+} \mathrm{T}$ cells (14).

The OX40L-DC-stimulated NKT cells may alter the tumor microenvironment, rendering it favorable for $\mathrm{CD}^{+} \mathrm{T}$ cell- and NK cell-mediated cytotoxicity. These NKT cells may also lead to a quantitative and/or qualitative reduction in the numbers of immune suppressive cells such as $\mathrm{CD}^{+}{ }^{+} \mathrm{Foxp}^{+}$Tregs. The local NKT activation, local inflammation, and local infiltration of lymphocytes (CD4 ${ }^{+}$and $\mathrm{CD}^{+} \mathrm{T}$ cells as well as NK cells) may be required for the effects of this OX40L-DC therapy. In future studies, it would be interesting to ask whether this approach could be applied to treat metastatic disease. Specifically, is a systemic response elicited, or is the response limited to the tumor into which the DCs are injected?

\section{New therapeutics for NKT cell-based cancer therapy}

In the clinic, disseminated disease may render intratumoral injection of OX40L-DCs to every tumor site impossible. Targeted delivery of immune activators such as OX40 agonists may represent a promising direction for new drug development. This approach is not without clinical precedent. Bifunctional conjugates of immune activators and tumor-binding antibodies, such as the anti-GD2-mAb-IL-2 fusion protein (15), have shown success in curing solid tumors in preclinical studies and already passed phase I clinical trials, proven to be safe and immunologically active. The efficacy of these drugs to cause objective responses of human cancer are being evaluated in phase II trials.

A new trend in both translational and clinical research is to combine immune activators that stimulate different cellular subsets, with the goal being to obtain synergistic effects and enhanced antitumor efficacy. Teng et al. recently published the "NKTMab" therapy method involving combined treatment with $\alpha$-GalCer and mAbs (16). Combination of these agents and approaches might be considered when designing new clinical trials.

Combination of immune activators and chemotherapeutics may also offer a mechanism to enhance the efficacy of either modality alone. Activating the adaptive immune system via tumor-specific antigens, immunotherapy might provide a layer of specificity and minimize the inherent toxicity of currently employed chemotherapy drugs. NKT cells may provide an ideal target for these combined approaches. They possess a unique role in bridging innate and adaptive immunity, they demonstrate potent activation of DCs, and their agonists have a large therapeutic window. These properties may allow this enigmatic lymphocyte population to be harnessed in cancer therapy.

\section{Acknowledgments}

I thank Alexander Gelbard, Chen Dong, Xin Lin, Patrick Hwu, and Yong-Jun Liu for their help and advice.
Address correspondence to: Dapeng Zhou, Department of Melanoma Medical Oncology, Division of Cancer Medicine, University of Texas MD Anderson Cancer Center, 1515 Holcombe Boulevard, Houston, Texas 77030, USA. Phone: (713) 792-3134; Fax: (713) 563-3424; E-mail: dzhou@mdanderson.org.

1. Watts, T.H., et al. 2005. TNF/TNFR family members in costimulation of T cell responses. Annu. Rev. Immunol. 23:23-68.

2. Melero, I., Hervas-Stubbs, S., Glennie, M., Pardoll, D.M., and Chen, L. 2007. Immunostimulatory monoclonal antibodies for cancer therapy. Nat. Rev. Cancer. 7:95-106.

3. Song, A., Song, J., Tang, X., and Croft, M. 2007. Cooperation between CD4 and CD8 T cells for anti-tumor activity is enhanced by OX40 signals. Eur. J. Immunol. 37:1224-1232.

4. Lee, S.J., et al. 2004. 4-1BB and OX40 dual costimulation synergistically stimulate primary specific CD8 T cells for robust effector function. J. Immunol. 173:3002-3012.

5. Murata, S., et al. 2006. OX40 costimulation synergizes with GM-CSF whole-cell vaccination to overcome established CD8 + T cell tolerance to an endogenous tumor antigen. J. Immunol. 176:974-983.

6. Zaini, J., et al. 2007. OX40 ligand expressed by DCs costimulates NKT and CD4 ${ }^{+}$Th cell antitumor immunity in mice. J. Clin. Invest. 117:3330-3338. doi:10.1172/JCI32693.

7. Smyth, M.J., Cretney, E., Kershaw, M.H., and Hayakawa, Y. 2004. Cytokines in cancer immunity and immunotherapy. Immunol. Rev. 202:275-293.

8. Song, L., et al. 2007. Oncogene MYCN regulates localization of NKT cells to the site of disease in neuroblastoma. J. Clin. Invest. 117:2702-2712. doi:10.1172/JCI30751.

9. Kawano, T., et al. 1997. CD1d-restricted and TCRmediated activation of valpha14 NKT cells by glycosylceramides. Science. 278:1626-1629.

10. Fujii, S., Liu, K., Smith, C., Bonito, A.J., and Steinman, R.M. 2004. The linkage of innate to adaptive immunity via maturing dendritic cells in vivo requires $\mathrm{CD} 40$ ligation in addition to antigen presentation and CD80/86 costimulation. J. Exp. Med. 199:1607-1618

11. Yu, Q., et al. 2006. OX40 ligation of CD4+ T cells enhances virus-specific CD8 $+\mathrm{T}$ cell memory responses independently of IL-2 and CD4+ T regulatory cell inhibition. J. Immunol. 176:2486-2495.

12. Ruby, C.E., Redmond, W.L., Haley, D., and Weinberg, A.D. 2007. Anti-OX40 stimulation in vivo enhances $\mathrm{CD} 8+$ memory $\mathrm{T}$ cell survival and significantly increases recall responses. Eur. J. Immunol. 37:157-166.

13. Serghides, L., et al. 2005. Evaluation of OX40 ligand as a costimulator of human antiviral memory CD8 T cell responses: comparison with B7.1 and 4-1BBL. J. Immunol. 175:6368-6377.

14. Zingoni, A., et al. 2004. Cross-talk between activated human NK cells and CD4+ T cells via OX40-OX40 ligand interactions. J. Immunol. 173:3716-3724.

15. Osenga, K.L., et al. 2006. A phase I clinical trial of the hu14.18-IL2 (EMD 273063) as a treatment for children with refractory or recurrent neuroblastoma and melanoma: a study of the Children's Oncology Group. Clin. Cancer Res. 12:1750-1759.

16. Teng, M.W., et al. 2007. Combined natural killer Tcell based immunotherapy eradicates established tumors in mice. Cancer Res. 67:7495-7504. 